\title{
Low-acceleration dwarf galaxies as tests of quantised inertia.
}

\author{
M.E. McCulloch*
}

March 6, 2017

\begin{abstract}
Dwarf satellite galaxies of the Milky Way appear to be gravitationally bound, but their stars' orbital motion seems too fast to allow this given their visible mass. This is akin to the larger-scale galaxy rotation problem. In this paper, a modification of inertia called quantised inertia or MiHsC (Modified inertia due to a Hubble-scale Casimir effect) which correctly predicts larger galaxy rotations without dark matter is tested on eleven dwarf satellite galaxies of the Milky Way, for which mass and velocity data are available. Quantised inertia slightly outperforms MoND (Modified Newtonian Dynamics) in predicting the velocity dispersion of these systems, and has the fundamental advantage over MoND that it does not need an adjustable parameter.
\end{abstract}

Keywords: celestial mechanics.

\section{Introduction}

The new Panoramic Survey Telescope and Rapid Response system (Pan-STARRS) and other surveys have recently discovered many new satellite galaxies of the Milky Way with luminosities of less than $10^{4} M_{\odot}$. The details of eleven of the systems for which both the visible mass and the orbital velocity are available are shown in Table 1 using data from (Laevens et al., 2015, Kirby et al., 2015, Simon et al., 2010, Simon and Geha, 2009, Martin et al., 2008, Koposov et al., 2011, Aden et al., 2009, Ibata et al., 2006 and Kleyna et al., 2005). The first column shows the system's name. The second column is its luminosity (in the visible band). The third column is the visible mass (determined by assuming the stars are of type K0, with a mass/light ratio of 1.95). The uncertainty in this mass is roughly a factor of two. The fourth column is the half-light radius $\left(r_{h l}\right)$ of the system. The fifth column is the observed velocity dispersion (Kirby et al., 2015 and Simon et al., 2010) and column six shows the maximum orbital

*Plymouth University, Plymouth, PL4 8AA. mike.mcculloch@plymouth.ac.uk 
speed $(v)$ of the stars consistent with a gravitationally bound state assuming Newtonian dynamics, ie: $v=\sqrt{G M / r}$. It is clear that assuming standard dynamics, all of these systems are orbiting far too fast to be gravitationally bound (although it has been shown that for Segue-1, Bootes-II, Leo IV and Hercules there is a small chance that the excess velocity dispersions could be due to the presence of binary stars, McConnachie and Côté, 2010).

These anomalies are similar to the galaxy rotation or galaxy cluster missing mass problem (Zwicky, 1937, Rubin and Ford, 1970) in which the outer stars of galaxies also show velocities too large to be bound by the gravitational pull of the galaxies' visible matter. In galaxies and galaxy clusters this is typically corrected ad hoc by adding dark matter to bind the galaxy gravitationally, but in these dwarf galaxies the amount of dark matter that needs to be added to the systems is several hundred to several thousand times the visible matter (for Segue 1 and Triangulum II it is up to 2600 and 3600 times) and it is unsatisfactory to have a solution that must be added to each galaxy by a different arbitrary amount.

\begin{tabular}{|c|c|c|c|c|c|c|}
\hline System & $\begin{array}{c}\text { Luminosity } \\
L_{\odot}\end{array}$ & $\begin{array}{c}\text { Vis' mass, } \\
M_{\odot}\end{array}$ & $\begin{array}{c}r_{h l} \\
\mathrm{pc}\end{array}$ & $\begin{array}{c}\text { Observed } \sigma_{V} \\
\mathrm{~km} / \mathrm{s}\end{array}$ & $\begin{array}{c}v_{N e w t o n} \\
\mathrm{~km} / \mathrm{s}\end{array}$ & $v_{M o N D}$ \\
\hline \hline Triangulum-II & $450_{-225}^{+225}$ & 877.5 & 34 & $5.1_{-1.4}^{+4.1}$ & $0.34_{-0.2}^{+0.6}$ & $1.9_{-0.5}^{+0.7}$ \\
\hline Segue-1 & $335_{-185}^{+235}$ & 653.25 & 30 & $3.7_{-1.1}^{+1.4}$ & $0.31_{-0.2}^{+0.6}$ & $1.8_{-0.6}^{+0.6}$ \\
\hline Ursa Major 2 & $4000_{-1900}^{+1800}$ & 7800 & 140 & $6.7_{-1.4}^{+1.4}$ & $0.5_{-0.3}^{+0.9}$ & $3.3_{-1.0}^{+1.1}$ \\
\hline Coma Berenices & $3700_{-1700}^{+1800}$ & 7215 & 70 & $4.6_{-0.8}^{+0.8}$ & $0.68_{-0.4}^{+1.3}$ & $3.3_{-0.9}^{+1.0}$ \\
\hline Bootes 2 & $1000_{-800}^{+800}$ & 1950 & 51 & $10.5_{-7.4}^{+7.4}$ & $0.41_{-0.1}^{+0.8}$ & $2.4_{-1.1}^{+0.9}$ \\
\hline Bootes 1 & $30000_{-6000}^{+6000}$ & 58500 & 242 & $5.7_{-3.3}^{+3.3}$ & $1.04_{-0.7}^{+1.6}$ & $5.5_{-1.1}^{+1.4}$ \\
\hline Canes Venatici 2 & $7900_{-3700}^{+34000}$ & 15,405 & 74 & $4.6_{-1}^{+1}$ & $0.96_{-0.5}^{+1.6}$ & $4.0_{-1.1}^{+1.1}$ \\
\hline Leo IV & $8700_{-4700}^{+4400}$ & 16,965 & 116 & $3.3_{-1.7}^{+1.7}$ & $0.81_{-0.4}^{+1.4}$ & $4.1_{-1.3}^{+1.3}$ \\
\hline Canes Venetici 1 & $200000_{-0}^{+100000}$ & 448,500 & 564 & $13.9_{-2.5}^{+3.2}$ & $1.88_{-1.3}^{+2.8}$ & $9.2_{-1.5}^{+2.1}$ \\
\hline Ursa Major 1 & $14000_{-4000}^{+4000}$ & 27,300 & 318 & $9.3_{-1.2}^{+11.7}$ & $0.62_{-0.4}^{+1}$ & $4.6_{-1.1}^{+1.2}$ \\
\hline Hercules & $36000_{-11000}^{+11000}$ & 70,200 & 330 & $5_{-1}^{+1}$ & $0.97_{-0.6}^{+1.6}$ & $5.8_{-1.4}^{+1.6}$ \\
\hline
\end{tabular}

Table 1. Physical parameters for the eleven dwarf galaxies considered here. The columns show the name of the system, its luminosity (with the range of error), its visible mass (with a error of a factor of two), its half-light radius, its observed velocity dispersion and the predictions of Newton/GR and MoND.

One alternative to dark matter is MoND (Modified Newtonian Dynamics) (Milgrom, 1983) and the more recent relativistic version of it by Bekenstein (2004) in which either the gravitational force on, or the inertial mass of, orbiting stars is varied for very low accelerations so that $\mu\left(g / a_{0}\right) g=g_{N}$ where $\mathrm{g}$ is the total acceleration, $g_{N}$ is the Newtonian acceleration and $a_{0}$ is an adjustable parameter set typically to $a_{0}=1.2 \times 10^{-10} \mathrm{~m} / \mathrm{s}^{2}$ (Famaey and McGaugh, 2012). The so-called interpolation function varies but Famaey and Binney (2005) found that $\mu=x /(1+x)$ is successful. Using this value for $a_{0}$, the predicted maximum speeds $\left(v=\left(G M a_{0}\right)^{0.25}\right)$ are still too low, as shown in column seven in Table 1. Furthermore, MoND is an empirical hypothesis that has no physical model and it relies on its adjustable parameter $\left(a_{0}\right)$ being fitted to the data by hand, which is unsatisfactory since no justification is given for this parameter. 
Milgrom (1999) noticed that the Unruh temperature (heat radiation seen only by an accelerating object) behaves rather like the inertial mass in MoND, but suggested that since the Unruh radiation was isotropic it was unlikely to be the cause for inertia.

However, McCulloch $(2007,2013)$ showed that there was a way to achieve an inertial model with Unruh radiation. When an object accelerates, say, to the right, an information horizon forms to its left. If it is then assumed that the wavelengths of Unruh waves have to fit into the distance between the object and the horizon (with nodes at the horizon and object) then the Unruh radiation will be suppressed by the horizon in the direction opposite to the acceleration and, so it becomes anisotropic, pushing the object back against its initial acceleration. This models standard inertia (McCulloch, 2013, Gine and McCulloch, 2016). Furthermore, this model predicts that some of the Unruh radiation will also be suppressed, this time isotropically, by the distant Hubble horizon which will make this mechanism less efficient, reducing inertial mass in a new way for very low accelerations for which Unruh waves are very long (McCulloch, 2007). This model, called MiHsC (Modified inertia by a Hubble-scale Casimir effect) or quantised inertia modifies the standard inertial mass $(m)$ as follows:

$$
m_{i}=m\left(1-\frac{2 c^{2}}{|a| \Theta}\right)
$$

where $\mathrm{c}$ is the speed of light, $\Theta$ is the co-moving diameter of the observable universe $\left(8.8 \times 10^{26} \mathrm{~m}\right.$, Bars and Terning, 2009) and $|\mathrm{a}|$ is the magnitude of the acceleration of the object relative to surrounding matter. Eq. 1 predicts that for terrestrial accelerations (eg: $9.8 \mathrm{~m} / \mathrm{s}^{2}$ ) the second term in the bracket is tiny and standard inertia is recovered, but in environments where the mutual acceleration is of order $10^{-10} \mathrm{~m} / \mathrm{s}^{2}$, for example at the edges of galaxies or in dwarf galaxies, the second term becomes larger and the inertial mass decreases in a new way. This modification does not affect equivalence principle tests using torsions balances since the predicted inertial change is independent of the mass. One might question why only the acceleration of the whole star determines ' $a$ ' and not the huge accelerations within the star. The answer is that in quantised inertia, the random accelerations of the hot atoms in the stars cancel out: they produce Rindler horizons all around the star symmetrically so there is no net effect on dynamics, but the small acceleration component that all the atoms jointly have, produce a systematic directional effect, so the ' $a$ ' in Eq. 1 is that of the star as a whole. The acceleration of the dwarf galaxy itself with respect to the Milky Way (the External Field Effect) is not used in Eq. 1 since the horizons formed by this acceleration occur on the side of the dwarf away from the Milky Way and so affect the collective motion, but not the internal dynamics.

In this way quantised inertia explains galaxy rotation without the need for dark matter (McCulloch, 2012) because it reduces the inertial mass of outlying stars and allows them to be bound even by the gravity from the visible matter. It also explains the recently observed cosmic acceleration (McCulloch, 2010). 
These results are encouraging, but not conclusive, since more flexible theories like dark matter, dark energy or MoND can be fitted to the data. Note that quantised inertia predicts a formula similar to the 'simple' interpolation function of MoND $\mu=x /(1+x)$ for higher accelerations, but the critical acceleration $\left(a_{0}\right)$ in quantised inertia is predicted by the theory itself and does not have to be input. It is not known if quantised inertia is consistent with Solar system data or not. Although MoND has been severely constrained by Solar system tests (Iorio, 2008) quantised inertia is fundamentally different from MoND.

Dwarf galaxies are ideal tests because they represent systems in which the acceleration is extremely low so the effects of quantised inertia should be noticeable, and also the observed dynamics are so unexpected that the amounts of dark matter needed are extreme and less convincing.

\section{Method}

We can model a dwarf spheroidal galaxy by equating the gravitational force holding its stars together and the inertial force pulling it apart, as follows

$$
\frac{G M m}{r^{2}}=\frac{m_{i} v^{2}}{r}
$$

where $G$ is the gravitational constant, $M$ is the mass of the dwarf galaxy within a radius $r$, and $v$ is the orbital velocity at radius $r$. Here $m$ is the gravitational mass and $m_{i}$ is the inertial mass of individual stars. Since it is usually assumed that $m=m_{i}$ this produces the Newtonian result

$$
v=\sqrt{\frac{G M}{r}}
$$

Now we can try the same thing with MiHsC using a derivation similar to the one for full-sized galaxies in McCulloch (2012). Starting with Newton's second law and his gravity law

$$
F=m_{i} a=\frac{G M m}{r^{2}}
$$

where $M$ is the mass of the system, $m$ and $m_{i}$ are the gravitational and inertial masses of a star, no longer assumed to be equal, and $a$ is the acceleration of the star in its orbit. Replacing the inertial mass with that from MiHsC (Eq. 1) gives

$$
m\left(1-\frac{2 c^{2}}{|a| \Theta}\right) a=\frac{G M m}{r^{2}}
$$


The ' $a$ ' can be split into a component of orbital acceleration that is constant $\left(a=v^{2} / r\right)$ and one that varies due to inhomogeneities in the dwarf galaxy, called $a^{\prime}$, so

$$
\left(1-\frac{2 c^{2}}{\left(|a|+\left|a^{\prime}\right|\right) \Theta}\right) a=\frac{G M}{r^{2}}
$$

Multiplying through by $a+a^{\prime}$ gives

$$
\left(|a|+\left|a^{\prime}\right|-\frac{2 c^{2}}{\Theta}\right) a=\frac{G M\left(|a|+\left|a^{\prime}\right|\right)}{r^{2}}
$$

MiHsC predicts a minimum acceleration of $2 c^{2} / \Theta$. This occurs because as accelerations reduce, the wavelength of the Unruh waves seen by an orbiting star lengthen and a greater proportion of them are disallowed by the Hubble-scale Casimir effect (they do not fit exactly inside the cosmic horizon, so are disallowed). Therefore, as the inertial mass decreases it become easier for a star to be accelerated into an orbital bound trajectory even by the small amount of visible matter. A balance is predicted to occur at a minimum acceleration as mentioned above of $2 c^{2} / \Theta$ (see McCulloch, 2007). For an orbiting star the rotational acceleration is less than $a=2 c^{2} / \Theta$ so the residual acceleration must appear in the $a^{\prime}$ term. Therefore $\left|a^{\prime}\right|=2 c^{2} / \Theta$ and the second and third terms in eq. 7 cancel to leave

$$
a^{2}=\frac{G M\left(|a|+\left|a^{\prime}\right|\right)}{r^{2}}
$$

Now we know that $a<a^{\prime}$. So we can approximate this, with

$$
a^{2}=\frac{G M\left|a^{\prime}\right|}{r^{2}}
$$

Since $a=v^{2} / r$ and $\left|a^{\prime}\right|=2 c^{2} / \Theta$ then

$$
v^{4}=\frac{2 G M c^{2}}{\Theta}
$$

Therefore, in MiHsC / quantised inertia

$$
v=\left(\frac{2 G M c^{2}}{\Theta}\right)^{\frac{1}{4}}
$$

This formula is similar to the MoND formula $v=\left(G M a_{0}\right)^{\frac{1}{4}}$ except that it is based on a stated physical model whereas MoND has no specific model, and 
also quantised inertia has no need for an adjustable parameter $a_{0}$, which (see Eq.11) is predicted by the model itself to be $2 c^{2} / \Theta=2 \times 10^{-10} \mathrm{~m} / \mathrm{s}^{2}$ (though see also the slightly different result of Pickering, 2017). There is therefore no possibility of 'tuning' MiHsC to fit the data so the fact that it agrees with the data is more significant. Equation 11 is only valid at the outer edge of dwarf galaxies since a minimal acceleration has been assumed, so it is not possible to predict the rotation curve in this way.

\section{Results}

Table 2 and Figure 1 show the results. In Table 1, the first column shows the dwarf galaxy studied, the second column is the maximum possible orbital speed predicted for stability by Newton (assuming no dark matter). Column 3 is the maximum possible orbital speed from $\operatorname{MoND}\left(v=\left(G M a_{0}\right)^{0.25}\right)$ assuming an adjustable factor of $a_{0}=1.2 \times 10^{-10} \mathrm{~m} / \mathrm{s}^{2}$. Column 4 is the maximum possible orbital speed predicted by quantised inertia. The error bars have been calculated using the uncertainty in the mass from Table 1. For comparison the observed orbital velocities are shown in column 5 with their error bars (uncertainties) as given in the sources.

\begin{tabular}{|c|c|c|c|c|}
\hline System & $\begin{array}{c}\text { Newtonian } \\
\mathrm{km} / \mathrm{s}\end{array}$ & $\begin{array}{c}\text { MoND } \\
\mathrm{km} / \mathrm{s}\end{array}$ & $\begin{array}{c}\text { MiHsC } \\
\mathrm{km} / \mathrm{s}\end{array}$ & $\begin{array}{c}\text { Observed } \\
\mathbf{k m} / \mathbf{s}\end{array}$ \\
\hline \hline Triangulum-II & 0.34 & $1.9_{-0.5}^{+0.7}$ & $2.2_{-0.6}^{+0.7}$ & $5.1_{-1.4}^{+4.1}$ \\
\hline Segue-1 & 0.31 & $1.8_{-0.6}^{+0.6}$ & $2.1_{-0.7}^{+0.7}$ & $3.7_{-1.1}^{+1.4}$ \\
\hline Ursa Major II & 0.50 & $3.3_{-1.0}^{+1.1}$ & $3.8_{-1.1}^{+1.1}$ & $6.7_{-1.4}^{+1.4}$ \\
\hline Coma Berenices & 0.68 & $3.3_{-0.9}^{+1.0}$ & $3.8_{-1.1}^{+1.2}$ & $4.6_{-0.8}^{+0.8}$ \\
\hline Bootes 2 & 0.41 & $2.4_{-1.1}^{+0.9}$ & $2.7_{-1.2}^{+1.0}$ & $10.5_{-7.4}^{+7.4}$ \\
\hline Bootes 1 & 1.04 & $5.5_{-1.1}^{+1.4}$ & $6.3_{-1.3}^{+1.6}$ & $5.7_{-3.3}^{+3.3}$ \\
\hline Canes Venatici 2 & 0.96 & $4.0_{-1.1}^{+1.1}$ & $4.5_{-1.2}^{+1.4}$ & $4.6_{-1.0}^{+1.0}$ \\
\hline Leo IV & 0.81 & $4.1_{-1.3}^{+1.3}$ & $4.6_{-1.4}^{+1.5}$ & $3.3_{-1.7}^{+1.7}$ \\
\hline Canes Venatici 1 & 1.88 & $9.2_{-1.5}^{+2.1}$ & $10.5_{-1.7}^{+2,4}$ & $13.9_{-2.5}^{+3.2}$ \\
\hline Ursa Major 1 & 0.62 & $4.6_{-1.1}^{+1.2}$ & $5.2_{-1.2}^{+1.4}$ & $9.3_{-1.2}^{+1.7}$ \\
\hline Hercules & 0.97 & $5.8_{-1.4}^{+1.6}$ & $6.6_{-1.5}^{+1.8}$ & $5.0_{-1.0}^{+1.0}$ \\
\hline
\end{tabular}

Table 2. The velocity dispersion predicted by Newton/GR, MoND and quantised inertia $(\mathrm{MiHsC})$ and the observed velocity dispersions, for the eleven dwarf galaxies studied. The observations were obtained from: Laevens et al., 2015, Kirby et al., 2015, Simon et al., 2010, Simon and Geha, 2009, Martin et al., 2008, Koposov et al., 2011, Aden et al., 2009, Ibata et al., 2006, Kleyna et al., 2005. The data is also shown graphically in Figure 1.

The Newtonian velocities are an order of magnitude too low. As mentioned before, the Newtonian or general relativistic models are inadequate by themselves, and the dark matter hypothesis requires the addition of several hundred times as much dark matter as visible matter to enable these systems to be bound, an addition which has to be added differently and arbitrarily for each case and so 
is scientifically unsatisfactory.

MoND performs better although it still tends to underpredict the velocity dispersion. It was noted by Angus (2008) that MoND does predict a higher mass/light ratio (or underpredict the velocity) for some dwarfs. A possible reason given for this was an incorrect distance measurement or uncertainties in the structure of the dwarfs. It has also been pointed out that the more tidally-susceptible dwarfs tend to be underpredicted by MoND (McGaugh \& Wolf, 2010) so tidal interactions with the Milky Way may be a cause. The more general problem is that MoND relies on an adjustable parameter $\left(a_{0}\right)$ which is usually set by hand to be $1.2 \times 10^{-10} \mathrm{~m} / \mathrm{s}^{2}$ and for which no physical reason is given.

The predictions of quantised inertia $(\mathrm{MiHsC})$ are slightly closer to the data than MoND, and crucially MiHsC achieves this without an adjustable parameter. The root mean squares differences between the predictions and the observations for Newton/GR, MoND and MiHsC are 6.5, 3.6 and $3.2 \mathrm{~km} / \mathrm{s}$ respectively, so $\mathrm{MiHsC}$ performs slightly better than MoND for these cases. MiHsC also requires no tuning and has a single, specific physical model that also predicts anomalies on the laboratory scale, for example, the anomalous behaviour of the emdrive (see eg: White et al., 2016) is predicted by MiHsC (McCulloch, 2015), so it is easier to test for than dark matter or MoND. A good test would also be to look for Unruh radiation in highly accelerated systems.

It is worth mentioning that the MiHsC formula used here (Eq. 11) is identical to the one used by McCulloch (2012) to successfully predict the rotation of dwarf galaxies, spiral galaxies, and galaxy clusters without dark matter.

\section{Conclusion}

Milky Way dwarf satellite galaxies appear to be bound systems, but the orbital motion of their stars is too fast to allow this. The solution of adding dark matter is problematic for dwarfs since hundreds of times more dark matter than visible matter must be added to keep them bound, and the arbitrariness of this process is deeply unsatisfying.

The predictions of the Newtonian/GR, MoND and quantised inertia (MiHsC) theories were tested for eleven dwarf galaxies for which mass and velocity dispersion data were available. Quantised inertia $(\mathrm{MiHsC})$ performed slightly better than MoND in these cases, and has a great advantage over MoND in not needing an adjustable parameter.

\section{References}

Aden, D., et al., 2009. A photometric and spectrospopic study of the new dwarf spheroidal galaxy in Hercules. Astron. \& Astrophys., 506, 3, 1147-1168.

Angus, G.W., 2008. Dwarf spheroids in MoND. MNRAS, 387, 1481-1488. 
Bars, I, J. Terning, 2009. Extra dimensions in space and time. Springer.

Bekenstein, J.D, 2004. Relativistic gravitation theory for the MOND paradigm. Phys. Rev. D., 70, 083509.

Famaey, B. and Binney J., 2005. MNRAS, 363, 603.

Famaey, B. and S.S. McGaugh, 2012. Modified Newtonian dyanamics (MoND): phenomenology and relativistic extensions. Living Rev. Relativity, 15, 10.

Gine, J., and M.E. McCulloch, 2016. Inertial mass from Unruh temperatures. Modern Physics Letters A, 31, 17, 1650107.

Ibata et al., 2006. MNRAS, 373, L70.

Iorio, L., 2008. Constraining MoND with Solar system dynamics. J. Grav. Physics., 2, 1, 26-32.

Kirby, et al., 2015. Triangulum II: possibly a very dense ultra-faint dwarf galaxy. Astrophysics Journal Letters, 814, L7.

Kleyna, J.T., et al., 2005. Ursa Major: a missing low-mass CDM halo? Astrophys. J., 630, L141-L144.

Koposov, S.E., et al., 2011. Accurate stellar kinematics at faint magnitudes: the Bootes-1 dwarf spheroidal galaxy. ApJ, 736: 146.

Laevens, B.P.M., et al., 2015. Astrophysical Journal Letters, 802, L18.

Martin, N.F., J.T.A de Jong, H-W., Rix, 2008. A comprehensive maximum likelihood analysis of the structural properties of fint Milky Way satellites. Astrophys. J., 684, 1075-1092.

McConnachie A.W., and P. Côté, 2010. Revisiting the influence of unidentified binaries on velocity dispersion measurements in ultra-faint stellar systems. Astrophys. J. - Letters., 722, L209-L214.

McCulloch, M.E., 2007. Modelling the Pioneer anomaly as modified inertia. MNRAS, 376, 338-342.

McCulloch, M.E., 2010. Minimum accelerations from quantised inertia, EPL, 90, 29001.

McCulloch, M.E., 2012: Testing quantised inertia on galactic scales. Astrophysics and Space Science, 342, 2, 575-578.

McCulloch, M.E., 2013. Inertia from an asymmetric Casimir effect. EPL, 101, 59001.

McCulloch, M.E., 2015. Testing quantised inertia on the emdrive. EPL, 111, 60005 .

McGaugh, S.S. and J Wolf, 2010. Local group dwarf spheroids: correlated deviations from the baryonic Tully-Fisher relation. ApJ, 722, 248-261.

Milgrom, M., 1983. The Astrophysical Journal, 270, 365-370.

Milgrom, M., 1999. Phys. Lett. A., 253, 273.

Pickering, K.A., 2017. The universe as a resonant cavity: a small step towards unification of MoND and MiHsC. AdAp, 1, 2, 247-249.

Rubin, V.C., W.K. Ford, 1970. The Astrophysical Journal, 159, 379. 
Simon, J.D, M. Geha, 2007. The kinematics of ultra-faint Milky Way satellites. Astrophys. J., 670, 313-331.

Simon, J.D., et al., 2010. A complete spectroscopic survey of the Milky Way satellite Segue-1: the darkest galaxy. The Astrophysical Journal, 733, 46.

White, H., March, P., Lawrence, J., Vera, J., Sylvester, A., Brady, D., Bailey, P., 2016. Journal of Propulsion and Power.

Zwicky, F., 1937. On the masses of nebulae and clusters of nebulae. Astrophysical Journal, 86, 217.

\section{Figures}

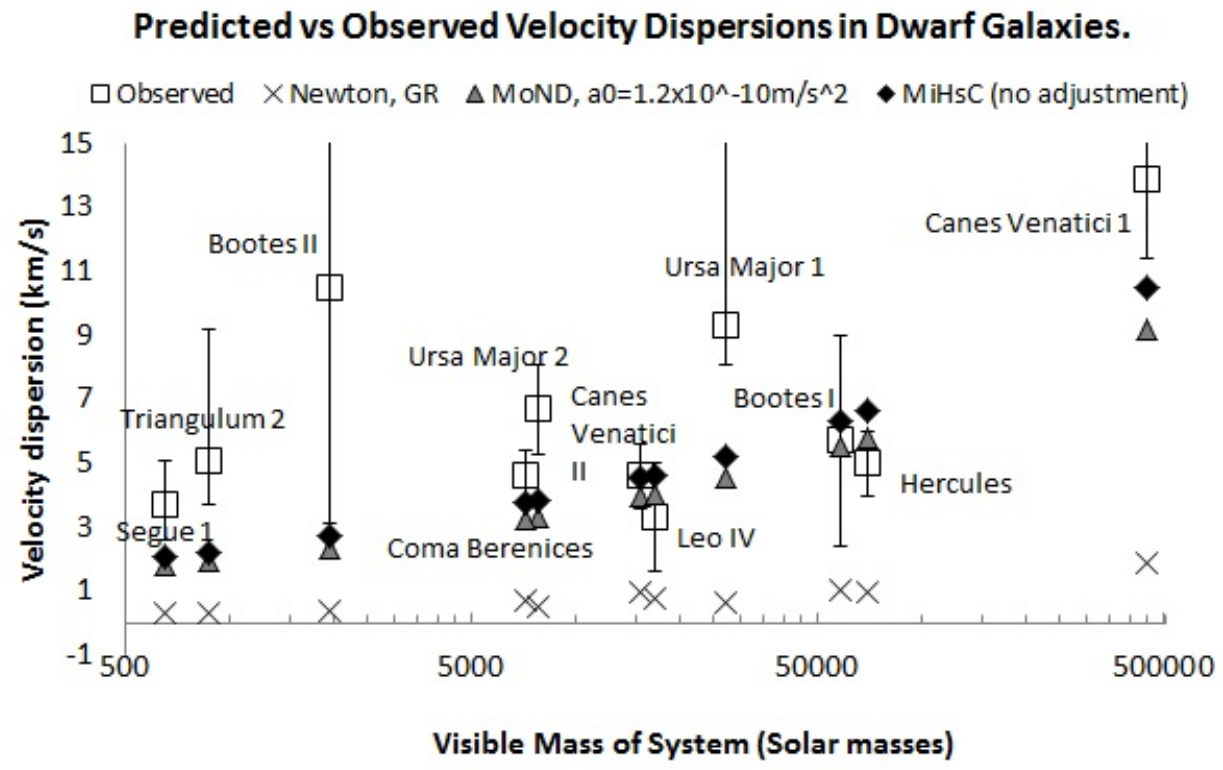

Figure 1. A comparison of the observed and predicted velocity dispersion of eleven of the Milky Way's dwarf galaxies. The $\mathrm{x}$ axis shows the systems' mass and the $y$ axis shows the velocity dispersion. The observations are shown by the open squares with vertical error bars and are taken from Laevens et al., 2015, Kirby et al., 2015, Simon et al., 2010, Simon and Geha, 2009, Martin et al., 2008, Koposov et al., 2011, Aden et al., 2009, Ibata et al., 2006 and Kleyna et al., 2005. The Newtonian prediction is shown by the crosses, and is far too low. The predictions of MoND (with $a_{0}=1.2 \times 10^{-10} \mathrm{~m} / \mathrm{s}^{2}$ ) are shown by the grey triangles and those of quantised inertia/ $\mathrm{MiHsC}$ by the black diamonds. MiHsC is slightly closer to the data than MoND, and MiHsC also needs no adjustable parameter. 